\title{
Novel algorithm for arrhythmogenic focus localization in patients with right ventricular outflow tract arrhythmias
}

\author{
Mariusz Pytkowski ${ }^{1}$, Aleksander Maciąg ${ }^{1}$, Maciej Sterliński ${ }^{1}$, \\ Agnieszka Jankowska ${ }^{1}$, Ilona Kowalik ${ }^{1}$, Michał M. Farkowski ${ }^{1}$, Rafał Kuteszko ${ }^{1}$, \\ Dariusz Zając ${ }^{1}$, Bohdan Firek ${ }^{1}$, Zbigniew Chmielak ${ }^{2}$, Hanna Szwed ${ }^{1}$ \\ ${ }^{1} 2^{\text {nd }}$ Department of Coronary Artery Disease, Institute of Cardiology, Warsaw, Poland \\ ${ }^{2}$ Department of Cardiology and Intervention Angiology, Institute of Cardiology, Warsaw, Poland
}

\begin{abstract}
Background: Previously presented new electrocardiography (ECG) algorithm for localization of arrhythmogenic focus ( $A F_{0}$ ) in right ventricular outflow tract (RVOT) was based on spontaneous arrhythmia $Q R S$ morphology analysis. The aim of this study was to estimate the clinical value of our RVOT algorithm in a prospective study.

Methods and results: Algorithm validation was made on 62 patients with RVOT arrhythmias (45 women), mean age $41.6 \pm 14.3$ years, scheduled for transcatheter ablation. Results of preablation ECG analysis with RVOT algorithm were matched with successful ablation sites and statistical indices: sensitivity (sens), specificity (spec), and positive and negative predictive values (PPV, NPV) were calculated for algorithm and for each of 9 RVOT zones (septal and free wall). An algorithm precisely localized AFo in 57 out of 62 patients (sens $91.3 \%$, spec 99\%, PPV 91\%, NPV 98.8\%). Sensitivity values for superior RVOT aspect (71\% patients) varied from $88 \%$ to $100 \%$, specificity from $95.9 \%$ to $100 \%$; PPV values from $85.7 \%$ to $100 \%$, NPV from $92.5 \%$ to $100 \%$. Although the total number of patients was relatively small in the 2 remaining $R V O T$ aspects (29\% patients) high values (sens, spec, PPV, NPV) were gained for intermediate and inferior zones.

Conclusions: On the basis of spontaneous arrhythmia QRS analysis, a novel algorithm was built for preablation localization of RVOT arrhythmia in 1 of the 9 RVOT zones. Prospective analysis of our ECG algorithm confirmed that it is a valuable tool to predict the site of successful ablation in patients with RVOT arrhythmias. (Cardiol J 2014; 21, 3: 284-292)
\end{abstract}

Key words: normal heart ventricular arrhythmias, RVOT, ECG algorithm

\section{Introduction}

Arrhythmia QRS morphology in patients with ventricular arrhythmias and no structural heart disease is connected with the localization of the arrhythmogenic focus [1-5]. The electrocardiographic (ECG) pattern of right ventricular outflow tract (RVOT) arrhythmias is typical and easily recognizable. In precordial leads those arrhythmias have left bundle branch block morphology (rS or QS waves in leads $V_{1}-V_{3}$ ) with inferior QRS axis in limb leads.

Endocardial mapping in patients with RVOT arrhythmias may be prolonged by extended step-

Address for correspondence: Mariusz Pytkowski, MD, $2^{\text {nd }}$ Department of Coronary Artery Disease, Institute of Cardiology, ul. Spartańska 1, 02-637 Warszawa, Poland, tel/fax: +48 2284495 10, e-mail: mpytkowski@ikard.pl 


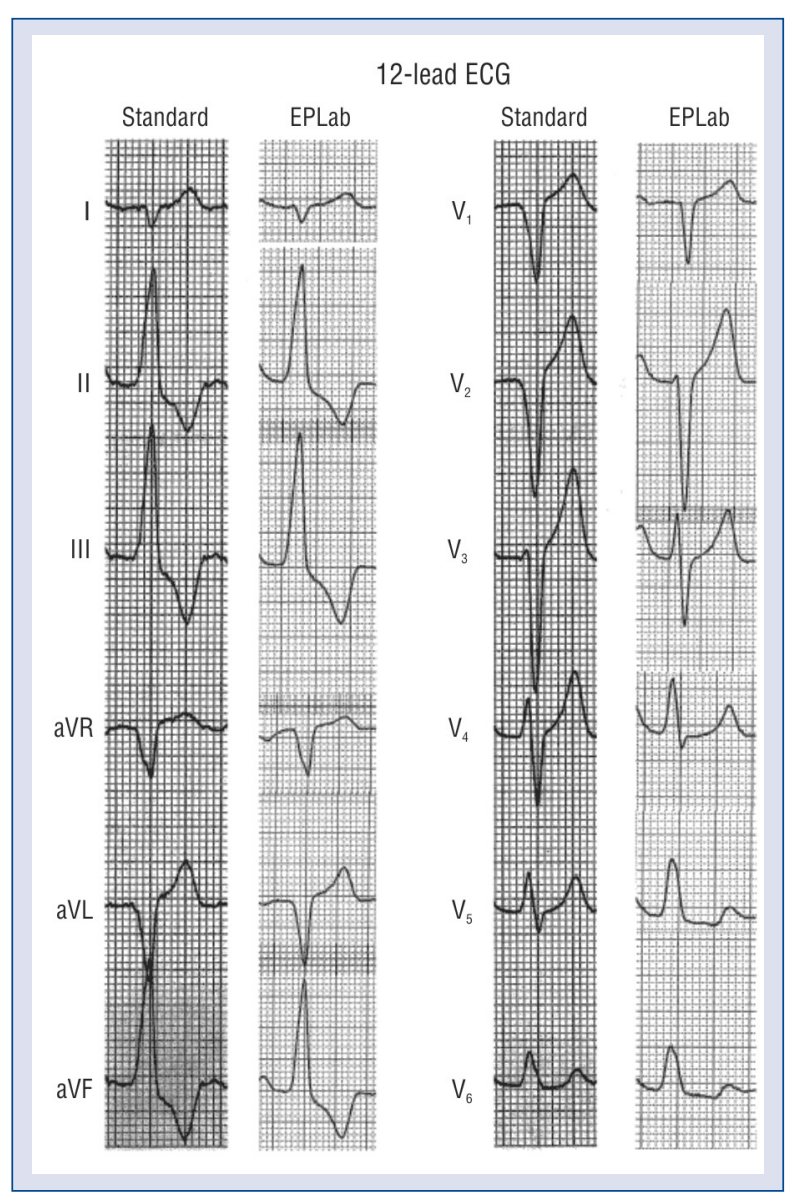

Figure 1. An example of typical differences of right ventricular outflow tract QRS complex in 12-lead electrocardiogram (ECG) obtained in the same patient in standard conditions and during electrophysiological (EP) study and ablation.

-by-step mapping of the relatively large RVOT area, so the preliminary localization of RVOT arrhythmogenic focus before radiofrequency catheter ablation (RFCA) is helpful and can shorten and simplify the ablation procedure. The basis of existing algorithms for RVOT arrhythmogenic foci localization is provided by studies on the comparative analysis of paced QRS complexes electrogram morphologies recorded during electrophysiology study (EPS) in certain RVOT regions [6-8]. During EPS additional ECG filters are often used. At the same time ECG electrodes placement (limb and precordial leads) differs from standards for 12-lead ECG (Fig. 1) $[9,10]$. This causes differences in QRS morphology and can lead to misinterpretations and mistakes in arrhythmogenic foci localizations on the basis of spontaneous arrhythmia 12-lead ECG recordings, which are typically included in the patient's medical records. To avoid such problems an algorithm for
RVOT arrhythmia foci localization based on spontaneous 12-lead QRS morphology was designed [11]. The aim of this single center prospective observational study was to evaluate the clinical value of our ECG algorithm for arrhythmogenic foci localization in RVOT.

\section{Methods}

\section{Patient group}

To begin with, from March 1998 to December 2001, 16 patients (14 women) with a mean age of $37 \pm 11.2$ years with RVOT arrhythmias were scheduled for ablation. Six out of the 16 patients had monomorphic ventricular tachycardia (VT) defined as 3 or more consecutive ventricular complexes, the remaining 10 patients suffered from frequent, resistant to antiarrhythmic drugs premature ventricular contractions (PVC). After the successful elimination of their arrhythmogenic foci an algorithm based on spontaneous arrhythmia QRS morphology was created to localize the arrhythmogenic foci in the RVOT.

In the next step validation of previously constructed algorithm was planned. From January 2002 to October 2010, 96 consecutive patients (72 women) in the mean age $43.6 \pm 13.5$ years, with normal heart ventricular arrhythmias were scheduled for transcatheter ablation. Clinical arrhythmias recorded in surface ECG showed QRS morphologies characteristic for arrhythmia arising from ventricular outflow tracts. From this group in 15 patients left ventricular outflow tract (LVOT) foci were highly possible on the basis of accepted ECG criteria. Finally, 81 patients with arrhythmia ECG morphology suggesting RVOT foci were included: inferior or normal axis in limb leads, left bundle branch morphology in precordial leads with negative QRS complex in $\mathrm{V}_{1}$ and $\mathrm{V}_{2}$. In 32 out of 81 patients with an early transition from negative to positive arrhythmia QRS in precordial leads $\left(\mathrm{r}<\mathrm{S}\right.$ or $\mathrm{r}=\mathrm{s}$ in $\mathrm{V}_{2}$ and $\mathrm{R}(\mathrm{r}) \geq \mathrm{S}(\mathrm{s})$ in $\left.\mathrm{V}_{3}\right)$ RVOT or out of RVOT foci localization were considered. Forty one patients had monomorphic VT. PVC with frequent periods of bigeminy and trigeminy were the only indication for transcatheter ablation in the remaining 40 patients. In all patients no structural abnormalities were found in a physical examination, 12-lead ECG during sinus rhythm and echocardiography. In the first 50 patients from this series, coronary angiography was performed in male patients older than 40 , female patients older than 55 with no coronary artery stenosis or other coronary artery or heart malformation found. 


\section{Study design}

The study protocol was approved by the institutional ethics committee and was in full compliance with the Declaration of Helsinki. An original algorithm created on the basis of spontaneous arrhythmia QRS morphology analysis in patients with RVOT arrhythmias was tested prospectively on 81 patients to assess its accuracy in predicting the 1 from 9 RVOT zones as the optimal site for transcatheter ablation in RVOT. The analysis of spontaneous arrhythmia QRS morphology was carried out supposing that during the ablation procedure some of our patients would have arrhythmogenic foci outside RVOT: in LVOT including sinuses of Valsalva, at the pulmonary trunk or other epicardial localizations. The prediction of the RVOT arrhythmia localization was made by one of the investigators prior to EPS and ablation.

\section{Electrophysiological testing and ablation}

EPS and ablation were performed after obtaining written informed consents. An EP testing, mapping and transcatheter ablation procedures were concordant with previously presented EP/ /RFCA protocol [12].

The total efficacy of the procedure was defined as the complete elimination of both spontaneous and induced arrhythmias of the morphology identical with that of clinical arrhythmia. Residual arrhythmia was defined as $\leq 300 \mathrm{PVC} / 24 \mathrm{~h}$. During the long-term follow-up symptomatic recurrences of arrhythmia, as well as standard ECG and 24-h ECG Holter monitoring were analyzed.

\section{Presentation of ECG algorithm to localize arrhythmogenic focus in patients with RVOT arrhythmias}

Analysis was performed on the basis of arrhythmia QRS morphology recorded in surface 12-lead ECG. The QRS deflections were coded in upper case letters when their amplitude was $\geq 0.5 \mathrm{mV}$ and in lower cases letters ( $\mathrm{q}, \mathrm{r}, \mathrm{s})$ when $\mathrm{QRS}$ wave amplitude was smaller than $0.5 \mathrm{mV}$. Deflections with amplitude of $0.1 \mathrm{mV}$ were considered. The transitional zone in precordial leads was defined as a shift from negative to positive QRS polarity in 2 consecutive precordial leads (e.g. transition from QS(qs) or $\mathrm{r}<\mathrm{S}$ in $\mathrm{V}_{3}$ to $\mathrm{R}(\mathrm{r})$ or $\mathrm{R}>\mathrm{s}$ in $\mathrm{V}_{4}$ ). In the same way the transitional zone in precordial leads was also defined as $r(R)=s(S)$ in certain leads (e.g. $V_{4}$ ) with negative $Q R S$ polarity in the preceding lead $\left(\mathrm{V}_{3}\right)$ and positive $\mathrm{QRS}$ polarity in the following lead $\left(\mathrm{V}_{5}\right)$. RVOT free wall sites were recognized in patients with RR' arrhythmia QRS

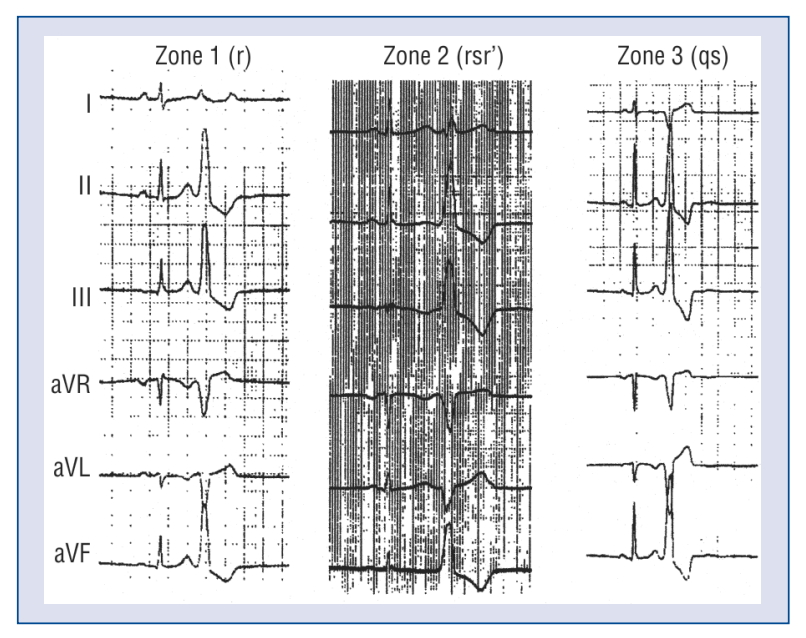

Figure 2. Arrhythmogenic focus localization in vertical zones of right ventricular outflow tract - differences of QRS morphology in lead I.

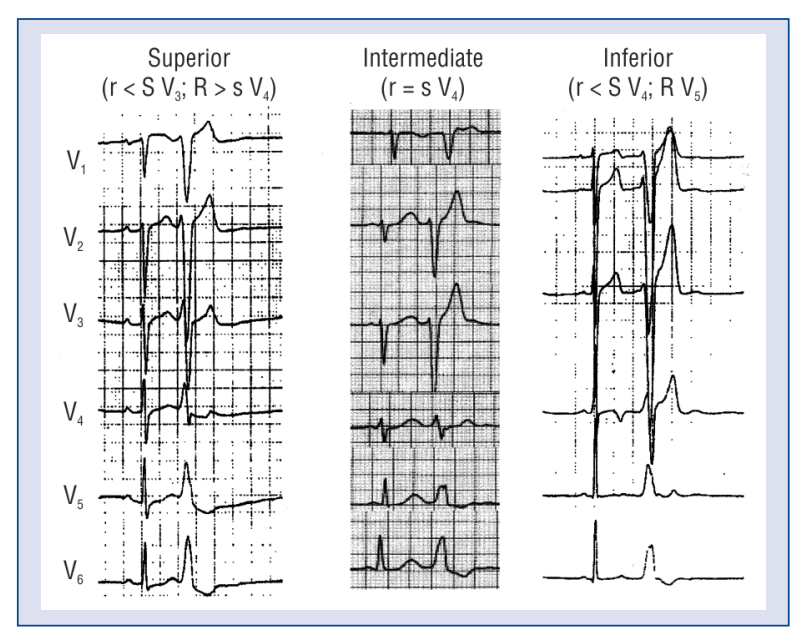

Figure 3. Arrhythmogenic focus localization in horizontal zones of right ventricular outflow tract - differences of QRS morphology in precordial leads.

morphology pattern in inferior ECG leads: II, III and $\mathrm{aVF}$, and there coexisted lower $\mathrm{R}$ wave amplitudes in those leads. On the basis of arrhythmia QRS morphology differences in limb lead I RVOT were divided into 3 vertical zones: 1, 2 and 3 (Fig. 2). Zone 3 is located in the anterior part of RVOT, zone 1 in the posterior part of RVOT and zone 2 in the middle part of RVOT between zones 1 and 3. Horizontal RVOT zones were formed on the basis of transition from negative arrhythmia QRS polarity to positive arrhythmia QRS polarity in precordial leads (Fig. 3). The horizontal intermediate zone because of the exact morphology in lead $V_{4}$ 


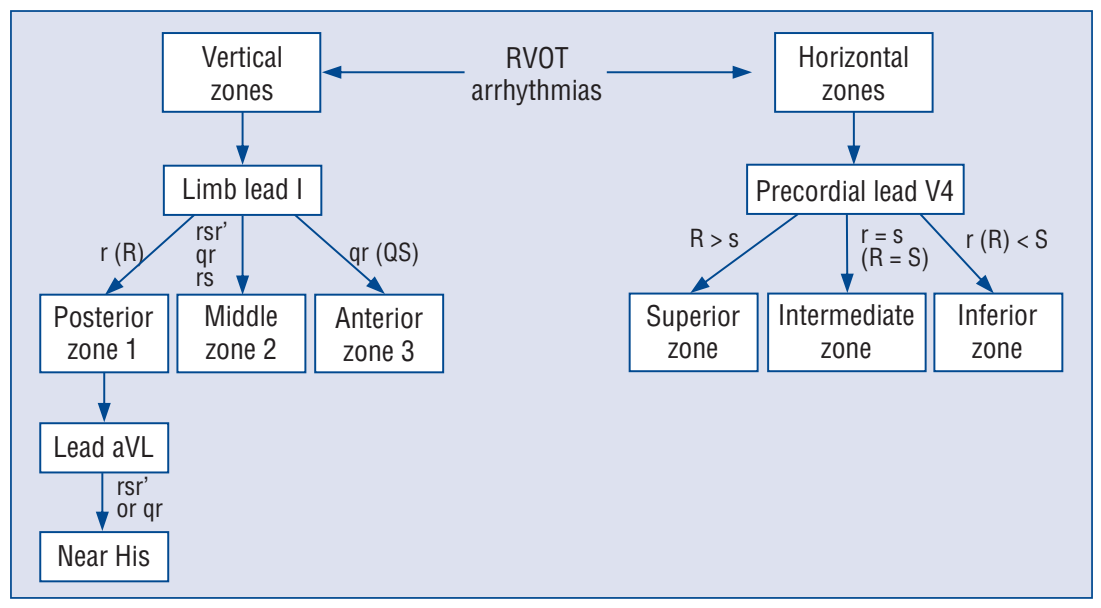

Figure 4. Algorithm for arrhythmia foci localization in patients with right ventricular outflow tract (RVOT) ventricular arrhythmias.

$\left(\mathrm{R}[\mathrm{r}]=\mathrm{S}[\mathrm{s}]\right.$ in $\left.\mathrm{V}_{4}\right)$ occupies a small portion of the median part of RVOT and separates the superior and inferior zones. 3 vertical and 3 horizontal zones divided RVOT into 9 sub-regions (Fig. 4).

\section{Predicting sites of successful ablation of arrhythmogenic focus in RVOT on the basis of own algorithm}

Spontaneous arrhythmia QRS morphologies were recorded and our ECG algorithm was implemented to localize RVOT arrhythmogenic focus in certain RVOT zones before ablation: septal and free wall. Those results were compared and were matched with sites of successful ablation. In patients with an early arrhythmia recurrence the last site of $\mathrm{RF}$ application that eliminated permanently RVOT arrhythmia was considered for further evaluation.

\section{Follow-up}

After the ablation procedure, before hospital discharge, $24 \mathrm{~h}$ ECG Holter was performed in all patients. During the follow-up at our cardiology clinic all patients were asked to undergo repeated 24-h Holter and 12-lead standard ECG at a 3-month visit after ablation. Sixty seven patients completed 3 -month follow-up visits at our outpatient clinic. The remaining 14 patients were interviewed by telephone and sent their 12-lead ECG and summaries of 24-h Holter recordings by fax. The next follow-up visit was made in medium and long term follow-ups from 9 to 26 months after the ablation procedure and this was possible in 63 out of the 81 patients ( 50 from 62 patients after transcatheter ablation in RVOT).

\section{Statistical analysis}

Statistical analyses were performed with SAS 9.2 Statistical Package. We calculated sensitivity, specificity, positive predictive values (PPV) and negative predictive values (NPV).

Recording of algorithm accuracy: sensitivity is defined as the percentage of patients with successful ablation in a certain RVOT zone in whom preablation evaluation by ECG algorithm revealed the same RVOT zone as the site of arrhythmogenic focus. Specificity is defined as the percentage of patients in whom certain RVOT zones were excluded in EPS and ablation and at the same time those RVOT zones were excluded in preablation evaluation by ECG algorithm. PPV represents the percentage of subjects with certain RVOT zones in ECG algorithm that were confirmed by successful ablation in the same RVOT zones. NPV represents the percentage of subjects in whom ECG algorithm excluded certain RVOT zones before RFCA, and that was confirmed during the EPS/ablation.

\section{Results}

\section{Transcatheter ablation}

Procedural success defined as complete elimination of clinical arrhythmia was achieved in 78 out of 81 patients. Ablation was not successful in 3 out of 32 patients with $\mathrm{r}<\mathrm{S}$ or $\mathrm{r}=\mathrm{s}$ in $\mathrm{V}_{2}$ and $\mathrm{R}(\mathrm{r}) \geq \mathrm{S}(\mathrm{s})$ in $\mathrm{V}_{3}$, in whom RVOT or out of RVOT localizations were considered before RFCA. In this group RVOT foci were confirmed and eliminated in 13 patients. In 16 patients from this group LVOT 
foci were found and ablated and those patients were excluded from analysis. In the remaining 49 patients with arrhythmia QRS morphology indicating RVOT localization, successful ablation confirmed RVOT foci in all patients. Finally, 62 patients (45 women) with RVOT arrhythmias were evaluated. In $55(88.7 \%)$ patients septal arrhythmogenic foci were ablated, in the remaining 7 (11.3\%) patients arrhythmogenic foci were located in RVOT free wall (Fig. 5).

Three patients from this group needed repeated ablation in RVOT due to symptoms recurrence confirmed by 24-h Holter monitoring in the first and third weeks after an initially successful RF ablation procedure. In these patients, repeated ablation in the same RVOT zones permanently eliminated the targeted arrhythmias. No complications related to RFCA were observed except for small local hematoma after right femoral artery puncture in 2 patients and after femoral veins puncture in 3 other patients. During ambulatory visits and transtelephonic contacts all patients were encouraged to contact with our center if the symptoms related to those experienced before ablation returned. Nobody from this group of patients claimed the symptoms recurrence after their last follow-up visit.

\section{Prospective evaluation of previously designed algorithm}

Arrhythmia QRS morphologies were recorded before ablation. Localization of arrhythmogenic foci, on the basis of our ECG algorithm and fluoroscopic (sites of RF ablations) localizations, lies in the same zones in 57 out of 62 patients (92\%). The differences were seen only in vertical RVOT zones (5 patients with septal arrhythmogenic foci). In 2 patients: no. 14 and no. 39 ECG algorithm localized the arrhythmogenic focus in zone 1 superior ( $\mathrm{r}$ wave in lead I; transitional zone in precordial leads $V_{3}-V_{4}$ ) but the successful $R F$ application was applied in zone 2 superior. In 1 patient (no. 58) ECG algorithm localized the arrhythmogenic focus in zone 3 superior (qs wave in lead I; transitional zone in precordial leads $\mathrm{V}_{3}-\mathrm{V}_{4}$ ) but the successful $\mathrm{RF}$ application was applied in zone 2 superior. In patient no. 42 an algorithm localization was 2 intermediate zone ( $\mathrm{rs}$ in lead I with $\mathrm{r}=\mathrm{s}$ in lead $\mathrm{V}_{4}$ ) but the ablation was made in 3 intermediate zone. In patient no. 17 an algorithm localized the arrhythmogenic focus in zone 1 inferior ( $\mathrm{r}$ wave in lead I; transitional zone in precordial leads $\mathrm{V}_{4}-\mathrm{V}_{6}$ ), but the ablation zone was 2 inferior. Full concordance was noted in ECG and fluoroscopic localizations

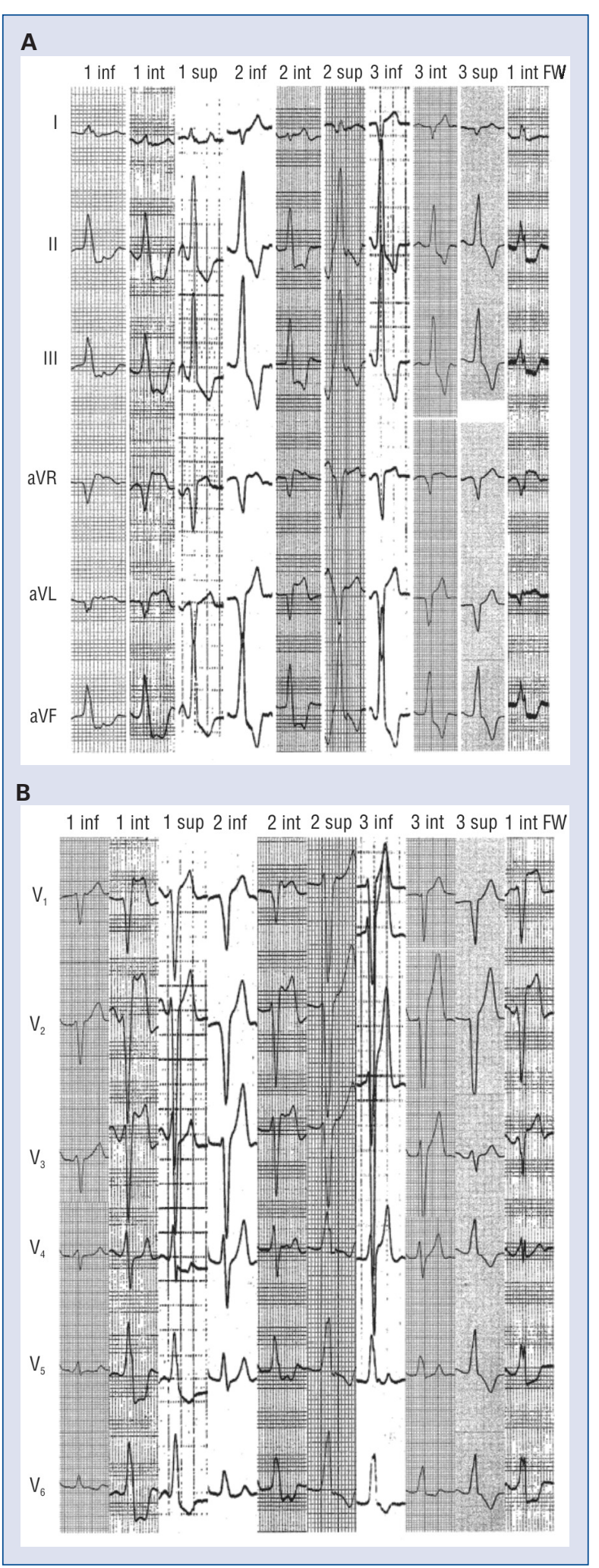

Figure 5. Right ventricular outflow tract (RVOT) QRS complex morphology changes in (A) vertical RVOT zones in limb leads and (B) horizontal RVOT zones in precordial leads, with an example of free wall (FW) focus in intermediate zone 1; RVOT horizontal zones: sup - superior; int - intermediate; inf - inferior; RVOT vertical zones: 1, 2, 3. 
of arrhythmogenic foci in horizontal zones. In 7 patients with free wall RVOT arrhythmogenic foci RVOT zones indicated by the algorithm were concordant with RF sites (Fig. 6).

\section{Algorithm validation. Sensitivity, specificity, positive and negative predictive values}

Arrhythmogenic foci in RVOT were located in superior aspect of RVOT (zones 1 superior, 2 superior and 3 superior) in 44 out of 62 patients (71\%). In the remaining 18 patients RVOT arrhythmogenic foci were located in medial and inferior part of RVOT septum and free wall (Fig. 6). Overall sensitivity for our algorithm was $91.3 \%$, specificity $99 \%$, PPV $91 \%$ and NPV $98.8 \%$. In addition, analysis of sensitivity, specificity, PPV and NPV were calculated for all 9 RVOT zones: septal and free wall. Sensitivity values for superior RVOT aspect varied from $88 \%$ to $100 \%$, specificity from $95.9 \%$ to $100 \%$; PPV from $85.7 \%$ to $100 \%$, NPV from $92.5 \%$ to $100 \%$. Although the total number of patients was relatively small, in the 2 remaining RVOT aspects (intermediate and inferior zones) high values were gained for those parts of RVOT: sensitivity values varied from $66.7 \%$ to $100 \%$, specificity from $98.3 \%$ to $100 \%$; PPV from $66.7 \%$ to $100 \%$, NPV from $98.3 \%$ to $100 \%$ (Table 1). Using accepted criteria we were able to present simple algorithm for normal heart ventricular arrhythmias analysis differentiating between RVOT and out-of RVOT arrhythmia localizations with detailed localization of RVOT arrhythmogenic foci in 1 of 9 RVOT zones (Fig. 7).

\section{Discussion}

Among a large group of arrhythmias with ECG bundle branch block pattern, those originating in RVOT can be the target for catheter ablation with procedure effectiveness of 90-95\% [2, 13-16]. Arrhythmogenic focus in RVOT is located in the majority of patients on the anterior or antero-lateral wall of sub-pulmonary valve right ventricular region $[8,15]$. Up to $34 \%$ of arrhythmogenic foci are located in the RVOT free wall [15]. The size of the septal part of RVOT is about $10 \mathrm{~cm}^{2}$ while focus size does not exceed a few square $\mathrm{mm}$ [17]. Thus mapping of the region without any earlier ECG-guided suggestions on arrhythmogenic focus localization may cause longer procedure and fluoroscopy times. There are 2 strategies to ablate RVOT arrhythmias. The first one is based on classic EP analysis with utilization of available ECG algorithms to localize arrhythmogenic

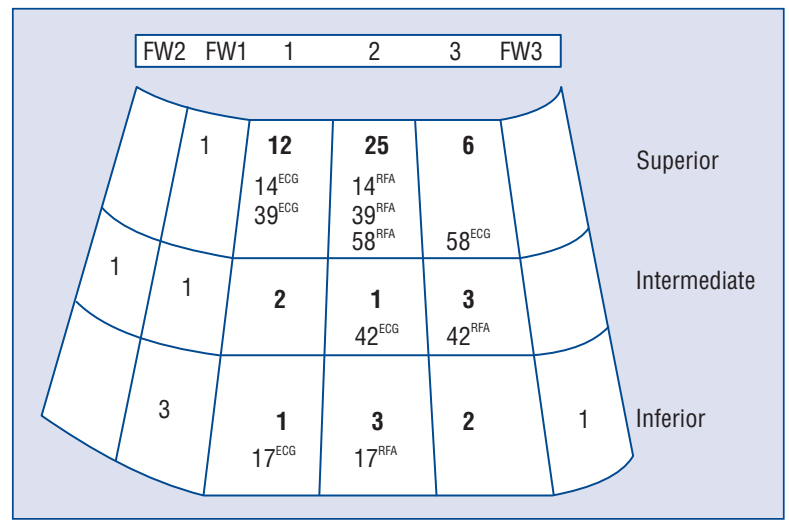

Figure 6. Localization of arrhythmogenic foci in right ventricular outflow tract (RVOT) in 62 patients using own created algorithm; 10, 23, 6 etc. number of patients in particular RVOT zones in whom arrhythmogenic foci were successfully ablated; $14,17,39,42$ and $58^{\mathrm{ECG}}-$ 5 patients in whom preablation ECG localization was different from ablation site; 14, 17, 39, 42 and 58 $8^{\text {RFA }}-$ sites of successful ablation in those patients; FW1, FW2, FW3 - free wall zones.

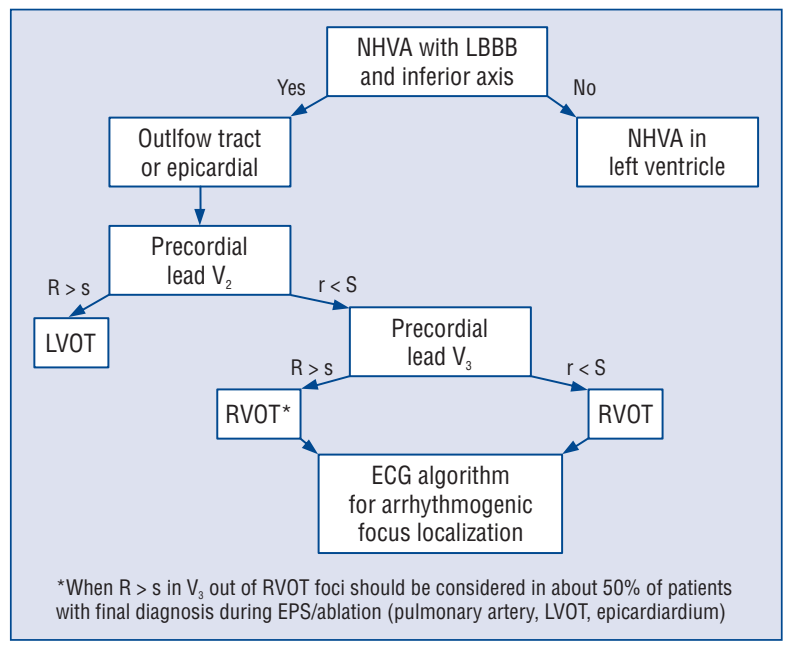

Figure 7. Algorithm for arrhythmia foci localization in patients with normal heart ventricular arrhythmias (NHVA); ECG - electrocardiogram, EPS - electrophysiological study; LBBB — left bundle branch block; LVOT — left ventricular outflow tract; RVOT — right ventricular outflow tract.

focus $[6,8]$. The second strategy utilizes 3 -dimensional (3D) mapping as 3D navigation systems, noncontact arrays and multielectrode basket catheters, to localize arrhythmogenic focus in RVOT $[18,19]$.

The majority of ECG algorithms for localization of arrhythmogenic focus in RVOT are based on 
Table 1. Prospective evaluation of electrocardiography (ECG) algorithm in 62 patients with right ventricular outflow tract (RVOT) arrhythmias (septal and free wall).

\begin{tabular}{|c|c|c|c|c|c|c|c|c|c|c|c|c|c|c|}
\hline \multirow{2}{*}{\multicolumn{2}{|c|}{$\begin{array}{l}\text { ECG algorithm } \\
\text { localization }\end{array}$}} & \multicolumn{9}{|c|}{ RVOT successful ablation site } & \multicolumn{4}{|c|}{ ECG algorithm } \\
\hline & & 1 & 2 & 3 & 1 & 2 & 3 & 1 & 2 & 3 & Sens & Spec & PPV & NPV \\
\hline 1 sup & 15 & 13 & 2 & & & & & & & & 100 & 95.9 & 86.7 & 100 \\
\hline 2 sup & 22 & & 22 & & & & & & & & 88.0 & 100 & 100 & 92.5 \\
\hline 3 sup & 7 & & 1 & 6 & & & & & & & 100 & 98.2 & 85.7 & 100 \\
\hline $1 \mathrm{int}$ & 3 & & & & 3 & & & & & & 100 & 100 & 100 & 100 \\
\hline $2 \mathrm{int}$ & 3 & & & & & 2 & 1 & & & & 100 & 98.3 & 66.7 & 100 \\
\hline 3 int & 2 & & & & & & 2 & & & & 66.7 & 100 & 100 & 98.3 \\
\hline $1 \mathrm{inf}$ & 5 & & & & & & & 4 & 1 & & 100 & 98.3 & 80 & 100 \\
\hline $2 \mathrm{inf}$ & 2 & & & & & & & & 2 & & 66.7 & 100 & 100 & 98.3 \\
\hline $3 \mathrm{inf}$ & 3 & & & & & & & & & 3 & 100 & 100 & 100 & 100 \\
\hline Total & 62 & 13 & 25 & 6 & 3 & 2 & 3 & 4 & 3 & 3 & $91.3 \pm 13.6$ & $99.0 \pm 1.3$ & $91.0 \pm 11.4$ & $98.8 \pm 2.3$ \\
\hline
\end{tabular}

Localizations of arrhythmogenic foci in RVOT: RVOT horizontal zones: sup — superior; int — intermediate; inf — inferior; RVOT vertical zones: 1, 2, 3; sens - sensitivity; spec — specificity; PPV — positive predictive value; NPV — negative predictive value

differences of the paced QRS complex in various sites of RVOT [4, 6, 20, 21]. Algorithms by Jadonath et al. [6], modified by Movsovitz et al. [8], were based on paced ECG analysis and classic EP mapping techniques. Algorithms by Dixit et al. [4] and Zhang et al. [18] utilized 3D mapping and paced QRS morphology analysis.

On the basis of a critical review of existing ECG algorithms based on pace mapping, constructed by Marchlinski's group, our own algorithm, based on arrhythmia QRS morphology, was designed in the years 1999-2001 and evaluated in a prospective study between 2002 and 2010 [4, 6, 8, 11]. The first algorithm from this author's group, constructed by Jadonath et al. [6], utilized pace mapping to show paced QRS morphology changes in various parts of RVOT in 11 patients with ventricular and supraventricular tachyarrhythmias. Following analyses made by Movsovitz, Garstenfeld and Dixit, introduced corrections to algorithm presented by Jadonath et al. [6]. A detailed analysis of those papers revealed that they were mostly retrospective, based on a relatively small number of patients. ECG signals analyzed by those authors were gained in EP Lab settings with nonstandard surface ECG leads positions and the application of additional filters [4, 5, 8]. Zhang et al. [18] published their own RVOT algorithm based on 3D mapping and QRS morphology analysis. Elaboration of the algorithm was based on retrospective analysis of 39 patients with RVOT arrhythmias and evaluation was made in the prospective study on 13 patients. Zhang et al. [18] used 3D mapping with utilization of Ensite system with noncontact array. At the same time, they used ECG obtained during EPS and ablation with all possible consequences mentioned above. Joshi et al. [15] presented a detailed analysis of all mapping techniques applied for RVOT arrhythmias. They confirmed our own and other authors' findings that "the vast majority of RVOT VT, both septal and free wall, originates from myocardium within $1-2 \mathrm{~cm}$ beneath the pulmonary valve". At the same time, a relatively large RVOT area depolarized in the first few milliseconds of ectopic activity, proving a limited value of 3D mapping in RVOT arrhythmias [17].

All presented papers were based on retrospective data analysis and relatively small groups of patients in prospective analysis. In only 1 paper, by Shima et al. [21], spontaneous arrhythmias were analyzed in 10 patients with RVOT arrhythmias. In that study, spontaneous arrhythmia ECGs were recorded during EPS with QRS complex morphology changes due to differences in standards of ambulatory 12-lead ECG which can lead to different conclusions. Only part of Shima's patients' group [21] underwent successful catheter ablation, so the real value of his data may be questioned.

Due to using originally recorded arrhythmia morphologies in 12-lead ECG we were able to avoid differences and artifacts caused by pacing during pace mapping, other ECG electrode placing on the patient's body during the EPS and ablation and using filters during the EPS (Fig. 1). The target site marked by our algorithm was con- 
firmed by successfully applied RF current with arrhythmogenic focus elimination in septal and free wall RVOT sites.

Our observations concerning limb lead I importance in localization of the arrhythmogenic focus in vertical plane of RVOT are very similar to those made by Movsovitz et al. [8]. We postulate the crucial role of the transitional zone in precordial leads in the prediction of arrhythmogenic focus localization in the horizontal plane of RVOT. We did not see very high $R$ waves in lead $V_{3}$ presented by Movsovitz et al. [8] at subpulmonic RVOT zone. We can speculate that those tall $\mathrm{R}$ waves were probably caused by the higher placing of ECG precordial leads during EPS and ablation. Although our RVOT horizontal zones are different in size, we used the RVOT division proposed by Jadonath et al. [15] and accepted by other authors [4, 6, 8]. The group of patients in our prospective analysis was sufficiently large enough to provide analysis of sensitivity, specificity, PPV and NPV separate for each RVOT segment of our ECG algorithm. The high value of our algorithm was confirmed by statistical values of overall sensitivity of $91 \%$ and specificity of $99 \%$ as statistical analysis of all RVOT zones. A high concordance of ECG algorithm locations and sites of successful ablation of RVOT arrhythmias legitimate the use of this algorithm in the process of pre-ablation target site analysis.

\section{Limitations of the study}

The intention of our group was to build an algorithm focused on RVOT. Therefore, other criteria and other algorithms should be applied to discriminate RVOT from outside RVOT arrhythmogenic foci $[2,3,15,22]$. As in other authors' series, in our analysis the majority of RVOT foci were located in the subpulmonary RVOT region. So, arrhythmogenic foci in other parts of RVOT, septal and free wall, are in the minority. In significant proportion of patients with outflow tracts ventricular arrhythmias an early transition from negative to positive arrhythmia QRS complex in precordial leads $\left(\mathrm{r}<\mathrm{S}\right.$ or $\mathrm{r}=\mathrm{s}$ in $\mathrm{V}_{2}$ and $\mathrm{R}(\mathrm{r})$ $\geq \mathrm{S}(\mathrm{s})$ in $\mathrm{V}_{3}$ ) can be found. Previous RVOT algorithms did not deal with those patients in whom several arrhythmogenic foci localizations are possible. Nearly $50 \%$ of them will be localized in RVOT, single in pulmonary trunk but significant proportion of those patients would be ablated from epicardial sites: can be reached from sinuses of Valsalva, via the coronary venous system (great cardiac vein and interventricular vein) or via the epicardium (epicardial puncture) [22]. We believe that using our algorithm in this specific group of patients' clinicians will get a message before ablation that they should be prepared for a much more complicated procedure, so they can avoid confusion concerning arrhythmogenic focus location during the procedure. Our study was made with classic EP techniques. Knowing the superiorities of 3D navigation system over the classic EP techniques we conclude that it is our study limitation.

\section{Conclusions}

On the basis of spontaneous arrhythmia QRS analysis novel algorithm was built for preablation localization of RVOT arrhythmia in 1 of 9 RVOT zones. Prospective analysis of our ECG algorithm confirmed that it is a valuable tool to predict the site of transcatheter ablation in patients with RVOT arrhythmias.

Funding: This work was supported by the Institute of Cardiology, Warsaw, Poland.

\section{Conflict of interest: none declared}

\section{References}

1. Ouyang F, Fotuhi P, Ho SJ et al. Repetitive monomorphic ventricular tachycardia originating from the aortic cusp (electrocardiographic characterization for guiding catheter ablation). J Am Coll Cardiol, 2002; 39: 500-508.

2. Ito $\mathrm{S}$, Tada $\mathrm{H}$, Naito $\mathrm{S}$ et al. Development and validation of an ECG algorithm for identifying the optimal site for idiopathic ventricular outflow tract tachycardia. J Cardiovasc Electrophysiol, 2003; 14: 1280-1286.

3. Cole CR, Marrouche NF, Natale A. Evaluation and management of ventricular outflow tract tachycardias. Cardiac Electrophysiol Rev, 2002; 6: 442-447.

4. Dixit S, Gerstenfeld EP, Callans DJ, Marchlinski FE. Electrocardiographic patterns of superior right ventricular outflow tract tachycardias: Distinguishing septal and free-wall sites of origin. J Cardiovasc Electrophysiol, 2003; 14: 1-7.

5. Gerstenfeld EP, Dixit S, Callans DJ, Rajawat Y, Rho R, Marchlinski FE. Quantitative comparison of spontaneous and paced 12-lead electrocardiogram during right ventricular outflow tract tachycardia. J Am Coll Cardiol, 2003; 41: 2046-2053.

6. Jadonath RL, Schwartzman DS, Preminger MW, Gottlieb CD, Marchlinski FE. Utility of the 12-lead electrogram in localizing the origin of right ventricular outflow tract tachycardia. Am Heart J, 1995; 130: 1107-1113.

7. Wilber DJ, Baerman J, Olshansky B, Kall J, Kopp D. Adenosine-sensitive tachycardia: Clinical characteristics and response to catheter ablation. Circulation, 1993; 87: 126-134.

8. Movsowitz C, Schwartzman D, Callans DJ et al. Idiopathic right ventricular outflow tract tachycardia: Narrowing the anatomic location for successful ablation. Am Heart J, 1996; 131: 930-936.

9. Kanagaratnam L, Tomassoni G, Schweikert R et al. Ventricular tachycardia arising from the aortic sinus of Valsalva: An under-recognized variant of left outflow tract ventricular tachycardia. J Am Coll Cardiol, 2001; 37: 1408-1414. 
10. Rodriguez L-M, Smeets JLRM, Timmermans C, Wellens HJJ. Predictors for successful ablation of right and left-sided idiopathic ventricular tachycardia. Am J Cardiol, 1997; 79: 309-314.

11. Pytkowski M, Maciag A, Sterlinski M et al. QRS morphology analysis to localize the arrhythmogenic focus in patients with right ventricular outflow tract arrhythmias. Abstract. Europace, 2002; 3 (suppl. A): A90,88/5.

12. Pytkowski M, Maciag A, Jankowska A et al. Quality of life improvement after radiofrequency catheter ablation of outflow tract ventricular arrhythmias in patients with structurally normal hearts. Acta Cardiol, 2012; 67: 153-159.

13. Sterlinski M, Pytkowski M, Maciag A et al. Radiofrequency catheter ablation in patients with focal ventricular arrhythmias. Kardiol Pol, 2002; 57: 1-7.

14. Lauribe P, Shah D, Jais P, Takahashi A, Haissaguerre M, Clementy J. Radiofrequency catheter ablation of drug refractory symptomatic ventricular ectopy: Short and long term results. PACE, 1999; 22:783-789.

15. Joshi S, Wilber DJ. Ablation of idiopathic ventricular outflow tract tachycardia: Current perspectives. J Cardiovasc Electrophysiol, 2005; 16 (suppl. 1): S52-S58.

16. Sadanaga T, Saeki K, Yoshimoto T, Funatsu Y, Miyazaki T. Repetitive monomorphic ventricular tachycardia of left coronary cusp origin. PACE, 1999; 22: 1553-1556.
17. Azegami K, Wilber DJ, Arruda M, Lin AC, Denman RA. Spatial resolution of pace mapping and activation mapping in patients with idiopathic right ventricular outflow tract tachycardia. J Cardiovasc Electrophysiol, 2005; 16: 823-829.

18. Zhang F, Chen M, Yang B et al. Electrocardiographic algorithm to identify the optimal target ablation site for idiopathic right ventricular outflow tract ventricular premature contraction. Europace, 2009; 11: 1214-1220.

19. Aiba T, Shimizu W, Taguchi A et al. Clinical usefulness of a multielectrode basket catheter for idiopathic ventricular tachycardia originating from right ventricular outflow tract. J Cardiovasc Electrophysiol, 2001; 12: 518-520.

20. Kamakura S, Shimizu W, Matsuo K et al. Localization of optimal ablation site of idiopathic ventricular tachycardia from right and left ventricular outflow tract by body surface ECG. Circulation, 1998; 98: 1525-1533.

21. Shima T, Ohnishi $Y$, Inoue $T$ et al. The relation between the pacing sites in the right ventricular outflow tract and QRS morphology in the 12-lead ECG. Jpn Circ J, 1998; 62: 399-404.

22. Tanner H, Hindricks G, Schirdewahn P et al. Outflow tract tachycardia with R/S transition in lead V3. Six different anatomic approaches for successful ablation. J Am Coll Cardiol, 2005; 45: 418-423. 